\title{
Evaluation of calcified carotid atheroma on panoramic radiographs and Doppler ultrasonography in an older population
}

\author{
Yusuf Atalay' \\ Fatih Asutay' \\ Kamil Serkan Agacayak ${ }^{2}$ \\ Mahmut Koparal ${ }^{3}$ \\ Fahri Adali ${ }^{4}$ \\ Belgin Gulsun² \\ 'Department of Oral and Maxillofacial \\ Surgery, Faculty of Dentistry, Afyon \\ Kocatepe University, Afyonkarahisar, \\ ${ }^{2}$ Department of Oral and Maxillofacial \\ Surgery, Faculty of Dentistry, Dicle \\ University, Diyarbakır, ${ }^{3}$ Department \\ of Oral and Maxillofacial Surgery, \\ Faculty of Dentistry, Adıyaman \\ University, Adıyaman, ${ }^{4}$ Department \\ of Cardiovascular Surgery, Faculty of \\ Medicine, Afyon Kocatepe University, \\ Afyonkarahisar, Turkey
}

This article was published in the following Dove Press journal:

Clinical Interventions in Aging

8 July 2015

Number of times this article has been viewed

\begin{abstract}
Aim: The aim of this study is to determine the reliability of panoramic radiograph (PR) as a screening tool for the detection of calcified carotid atheroma (CCA) by comparing it with Doppler ultrasonography (DU) examination. A second aim was to evaluate the relationship among CCA, systemic diseases, smoking, and body mass index in an older population.
\end{abstract}

Materials and methods: A total of 1,650 PRs of patients aged over 45 years (736 males and 914 females) were randomly selected. All the patients had been referred to the Faculty of Dentistry, Afyon Kocatepe University, Afyonkarahisar, Turkey, during 2013-2014 for routine PR screening. Medical data were collected from the archival records of the dental school. The patients were divided into two groups: Group A (study group), CCA findings were confirmed by DU ( $\mathrm{n}=59)$; and Group B (control group), CCA findings were not confirmed by DU ( $\mathrm{n}=34)$.

Results: Of the 1,650 individuals, 93 (5.63\%) were detected to have CCA on PR. The population consisted of 43 males and 50 females with mean age of $59.84 \pm 10.92$ years. No difference was determined in respect of CCA between the sexes $(P=0.745)$. There was a significant difference between Group A and Group B in respect of hypertension $(P=0.004)$. But there was no difference between Group A and Group B in respect of age $(P=0.495)$, BMI $(P=0.756)$, diabetes ( $P=0.168)$, and smoking $(P=0.482)$ distribution.

Conclusion: Although PR cannot be used as an initial diagnostic method when searching for CCA, dentists should be aware of CCA on a routine PR, particularly in older patients who may also have the risk factors of obesity, diabetes mellitus, hypertension, and smoking. Recognizing of CCA especially in hypertensive patients could potentially increase the length and quality of life for individuals.

Keywords: panoramic radiograph, calcified carotid atheroma, atherosclerosis, risk factors, ultrasonography

\section{Introduction}

Stroke remains one of the most leading causes of death and a main cause of long-time disability in most countries. ${ }^{1}$ Stroke is generally the result of embolus or thrombus delivered from a carotid atheromatous plaque, or more rarely due to hemodynamic changes arising from the considerable contraction of the carotid lumen. ${ }^{2}$ Calcified carotid atheroma (CCA) consists of a calcification plaque of cholesterol and fat within the carotid artery, and known risk factors include diabetes mellitus, hypertension, hyperlipidemia, obesity, and smoking. ${ }^{3}$

Although stroke can be avoided, a major challenge is to discover efficient methods to detect stroke-prone patients. Most noncardiogenic strokes happen as a result of

Correspondence: Yusuf Atalay Afyon Kocatepe University, Faculty of Dentistry, Department of Oral and Maxillofacial Surgery, 03030 Afyonkarahisar, Turkey Tel +90272 2167900 Ext 1038

Email dratalay@aku.edu.tr 
atheroma plaques in the internal carotid artery, calcification of which can be detected on panoramic radiograph (PR) ${ }^{4}$ Cohen et $\mathrm{al}^{5}$ thought PRs to be significant markers for following vascular events, and recommended that patients with CCA be referred for cerebrovascular and cardiovascular evaluation. On a routine PR, CCA can be detected primarily by the oral practitioner. Almost $5 \%$ of PRs of patients aged over 30 years show feasible CCA. ${ }^{6}$ This calcification may appear in the form of nodular, or a verticolinear radiopacity inferior to the angulus mandible, adjacent to the $\mathrm{C} 3-\mathrm{C} 4$ intervertebral junction. ${ }^{7-9}$ However, PR is restricted to the diagnosis of the atheroma and cannot identify its exact location or degree of occlusion. Diagnosis may be made by means of ultrasonography, as a noninvasive and comparatively cheap technique. ${ }^{6,10}$

Many studies report the usefulness of the PR for identifying stroke patients by relationship with systemic diseases, cervical radiographs, or ultrasonography analysis., ${ }^{5,11,12}$ But some studies were limited either by small sample sizes, or by not analyzing whether the carotid artery was actually calcified. ${ }^{12,13}$ There are not yet enough data confirming the relationship between the CCA shown on the PR and systemic diseases or smoking. Therefore, the present study was made to determine the reliability and prevalence of CCAs on PRs and relationship among systemic diseases, smoking, BMI and $\mathrm{CCA}$ in a group of patients aged over 45 years. As a result, careful evaluation of PRs could be lifesaving.

\section{Materials and methods Study population}

In this study, a total of 1,650 PRs of patients aged over 45 years (736 males and 914 females) were retrospectively, randomly selected. All the patients had been referred to the Faculty of Dentistry, Afyon Kocatepe University, Afyonkarahisar, Turkey, during 2013-2014 for routine PR screening, and had recent, complete, medical records. The patients were contacted and informed consent was obtained from all the participants. The PRs had originally been taken for routine patient clinical examination and not for the investigation of CCAs. Approval for the study was granted by the Afyon Kocatepe University Faculty of Medicine Ethics Committee.

Patients were excluded if they had cancer or other serious comorbidities (and were thus not suitable for study), a previous stroke, or transient ischemic attack, since the aim was to study patients without any previous cerebrovascular event. When atheromatous plaque was detected, certain information (age, sex, smoking habits, weight, height, medical history, and uni/bilateral CCA appearance) was recorded. A total of



Figure I A panoramic radiograph of a 76-year-old female. There were bilateral multiple radiopacities of suspected calcified carotid atheromas (white circles) (Group A, study group).

93 patients (43 male and 50 female, age range $45-84$ years) with CCA on PR were included for evaluation in the study (Figures 1 and 2). CCA was checked via Doppler ultrasonography (DU) in 93 patients (Figures 3 and 4).

The patients were divided into two groups as follows:

- Group A (study group): CCA findings were tested by DU and CCA was confirmed ( $\mathrm{n}=59)$.

- Group B (control group): CCA findings were tested by DU but presence of CCA was not confirmed ( $\mathrm{n}=34)$.

Hypertension was defined as current use of antihypertensive medication or systolic blood pressure of $>140 \mathrm{mmHg}$ and/or a diastolic blood pressure of $>90 \mathrm{mmHg}$. Diagnosis of patients with diabetes was made from fasting plasma glucose $>125 \mathrm{mg} / \mathrm{dL}$. Body mass index (BMI) was classified according to WHO criteria with normal weight: 18-25 $\mathrm{kg} / \mathrm{m}^{2}$; over weight, $25-30 \mathrm{~kg} / \mathrm{m}^{2}$; obese class 1 (moderate obese), $30-35 \mathrm{~kg} / \mathrm{m}^{2} .{ }^{14}$

\section{Panoramic radiography}

PRs were taken at the Faculty of Dentistry, Afyon Kocatepe University, Afyonkarahisar, Turkey, using the Morita Veraview IC-5 HD (Morita, Tokyo, Japan) panoramic X-ray systems. The range of exposure value was $1-7.5 \mathrm{~mA}$ and $60-70 \mathrm{kV}$

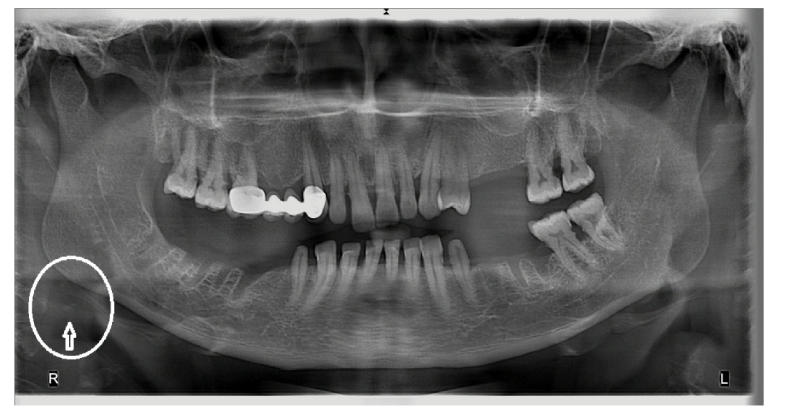

Figure 2 A panoramic radiograph of a 56-year-old female. There was unilateral radiopacity of suspected calcified carotid atheroma (white circle) (Group B, control group). 


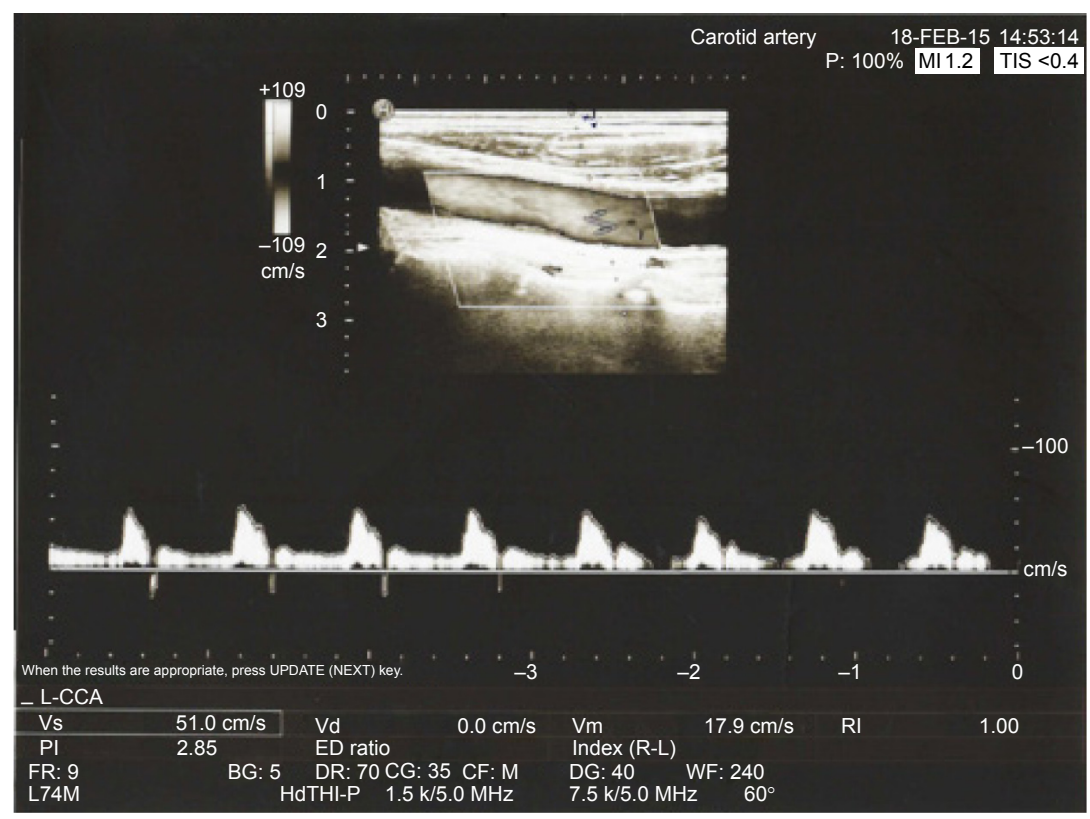

Figure 3 A Doppler ultrasound image of a 76-year-old female. There was an evident calcified carotid atheroma (Group A, study group).

depending on the patient's size. The PRs were examined by one of the authors. Using the differential diagnosis of CCA described by Friedlander ${ }^{15}$ when there was a radiopaque nodular mass independent of the hyoid bone adjacent to the cervical vertebrae at or below the $\mathrm{C} 3-\mathrm{C} 4$ intervertebral disc level, or the retromandibular area, generally at an angle of about $45^{\circ}$ from the angle of mandible, it was regarded as CCA. To minimize the discrepancy of observations, all PRs were evaluated by the same author.

\section{Doppler ultrasonography}

DU imaging studies of the carotid artery were performed at the Department of Radiology, Faculty of Medicine, Afyon Kocatepe University, Afyonkarahisar, Turkey, for all subjects to confirm the presence of CCA. All the ultrasound examinations were performed by the same radiologist, who was blinded to the findings on PR. Bilateral common carotid and proximal internal carotid arteries were examined with DU using a 7.5 MHz linear array transducer (Hitachi, Tokyo, Japan).

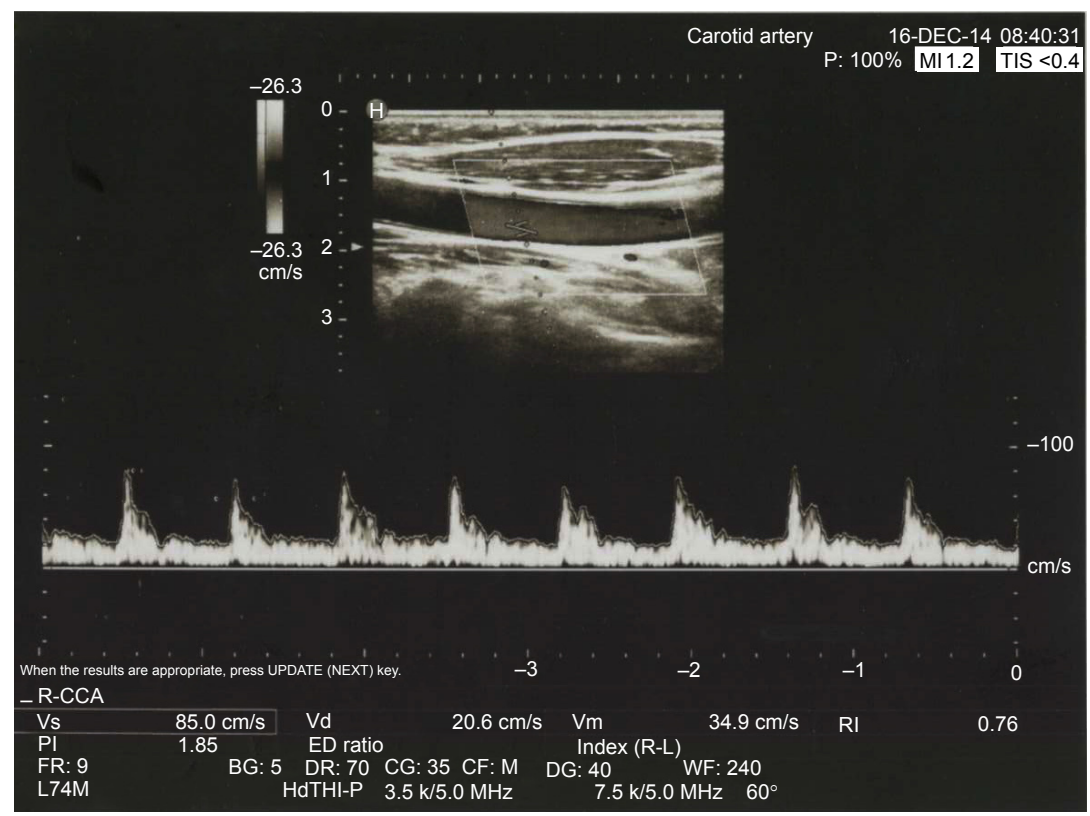

Figure 4 A Doppler ultrasound image of a 56-year-old female. There was no calcified carotid atheroma (Group B, control group). 


\section{Statistical analysis}

The prevalence and accuracy of CCA was calculated as the percentage of the total number of subjects. In the group comparisons of quantitative variables such as age and BMI, the Mann-Whitney $U$-test was used. In the evaluations of categorical data, the Pearson chi-square and the Fischer's exact tests were applied in the comparisons between groups. A value of $P<0.05$ was accepted as statistically significant. All analyses were conducted using the SPSS version 14.0 statistical program.

\section{Results}

The screened population consisted of $736(44.60 \%)$ males and $914(55.40 \%)$ females with an age range of 45-84 years at the time the PRs were taken. Of the 1,650 individuals, $93(5.63 \%)$ were detected to have CCA on PR. The population consisted of 43 males and 50 females with mean age of $59.84 \pm 10.92$ years (male: $58 \pm 10.15$ years, female: $61.12 \pm 11.49$ years). No difference was determined in respect of CCA between the sexes $(P=0.745)$.

In Group A, these CCA findings were confirmed by DU imaging as hyperechoic signals with distal acoustic shadow. This examination showed $63.44 \%$ accuracy for the detection of CCA using PR. The validated Group A comprised 59 patients: 28 males and 31 females with a mean age of $60.46 \pm 11.01$ years (male: $59.07 \pm 10.85$ years, female: $61.71 \pm 11.19$ years). No statistically significant difference was determined in respect of CCA between the sexes $(P=0.756)$.

In Group A, CCA was identified as bilateral in 18 males and ten females and unilateral in ten males (seven right side, three left side) and 21 females (12 right side, nine left side) (Table 1, Figure 5). No significant difference was found between right and left side CCA $(P=0.209)$. No statistically significant difference was determined in respect of left and right side CCA in both males and females (male, $P=0.206$; female, $P=0.513)$.

The systemic records of 59 patients in Group A were analyzed for the risk factors associated with CCA. Table 1 shows the relationship between medical history, smoking, BMI, and CCA. In Group A, 21 (12 male, nine female) subjects (35.59\%) reported hypertension, 16 (six male, ten female) subjects (27.11\%) reported diabetes mellitus, and 16 (14 male, two female) subjects $(27.11 \%)$ reported smoking (Figure 6). Of these 59 patients, $12(20.33 \%)$ were normal weight $(\mathrm{BMI}=18.5-25), 34(57.62 \%)$ were overweight $(\mathrm{BMI}=25-30)$, and ten $(16.94 \%)$ were moderately obese $(\mathrm{BMI}=30-35)$.

In Group B, verification of CCAs was tested by DU but no CCA was found. The 34 patients comprised 15 males and
19 females with a mean age of $58.76 \pm 10.84$ years (male: $57 \pm 8.9$ years, female: $60.16 \pm 12.21$ years). No statistically significant difference was determined in respect of CCA between the sexes $(P=0.493)$.

In Group B, CCA was identified as bilateral in three males and two females and unilateral in 12 males (six right side, six left side) and 17 females (eight right side, nine left side) (Table 2, Figure 5). No significant difference was determined between the right and the left sides of CCA $(P=0.853)$. No statistically significant difference was determined in respect of left and right side CCA in both males and females (male, $P=1.000$; female, $P=0.808$ ).

The systemic records of 34 patients in Group B were analyzed for the risk factors associated with CCA. The correlations between medical history, smoking, BMI, and CCAs are shown in Table 2. In Group B, three (one male, two female) subjects $(8.82 \%)$ reported hypertension, five (four male, one female) subjects (14.7\%) reported diabetes mellitus, and seven (six male, one female) subjects (20.58\%) reported smoking (Figure 6). Of these 34 patients, eleven (three male, eight female) $(32.35 \%)$ were normal weight $\left(\mathrm{BMI}=18.5-25 \mathrm{~kg} / \mathrm{m}^{2}\right), 19$ (eight male, eleven female) $(55.88 \%)$ were overweight $\left(\mathrm{BMI}=25-30 \mathrm{~kg} / \mathrm{m}^{2}\right)$, and four (four male, zero female) (11.76\%) were moderately obese (BMI $=30-35 \mathrm{~kg} / \mathrm{m}^{2}$ ).

A statistically significant difference was determined between Group A and Group B in respect of hypertension rates $(P=0.004)$. But there was no statistically significant difference between Group A and Group B in respect of age ( $P=0.495)$, BMI $(P=0.756)$, diabetes $(P=0.168)$, and smoking $(P=0.482)$ distribution (Figures 6 and 7).

A statistically significant difference was determined between the males and females in Group A and Group B in respect of BMI rates $(P=0.017, P=0.046)$ and smoking rates. $(P=0.001, P=0.013$ ) (Figures 6, 8, and 9). But there was no statistically significant difference between the males and females in Group A and Group B in respect of age distribution ( $P=0.327$, $P=0.444)$ and hypertension rates $(P=0.291, P=0.691)$.

A statistically significant difference was determined between the males and females in Group B in respect of diabetes rates $(P=0.08)$. No statistically significant difference was determined between the males and females in Group A in respect of diabetes rates $(P=0.35)$ (Figure 6).

\section{Discussion}

Our aim was to determine the reliability of PR as a screening tool for the detection of CCA by comparing it with DU examination. A second aim was to evaluate the relationship among CCA, systemic diseases, smoking, and BMI in an 
Table I The demographic and medical records of Group A

\begin{tabular}{|c|c|c|c|c|c|c|c|}
\hline Patient & Sex & Age (years) & Side & Hypertension & Diabetes & Smoking & BMI $\left(\mathrm{kg} / \mathrm{m}^{2}\right)$ \\
\hline I & M & 45 & $\mathrm{R}$ & HT & DM & $S>10$ & 27.7 \\
\hline 2 & $M$ & 55 & $R / L$ & $\mathrm{n} / \mathrm{d}$ & $\mathrm{n} / \mathrm{d}$ & $S>10$ & 28.1 \\
\hline 3 & $M$ & 49 & $\mathrm{R}$ & HT & $\mathrm{n} / \mathrm{d}$ & $\mathrm{n} / \mathrm{d}$ & 25.2 \\
\hline 4 & $M$ & 79 & $R / L$ & HT & $\mathrm{n} / \mathrm{d}$ & $S>10$ & 34.1 \\
\hline 5 & $M$ & 48 & $\mathrm{~L}$ & $\mathrm{n} / \mathrm{d}$ & DM & $\mathrm{n} / \mathrm{d}$ & 35 \\
\hline 6 & $M$ & 73 & $\mathrm{R}$ & $\mathrm{n} / \mathrm{d}$ & $\mathrm{n} / \mathrm{d}$ & $S>10$ & 27.7 \\
\hline 7 & $M$ & 56 & $R / L$ & HT & $\mathrm{n} / \mathrm{d}$ & $n / d$ & 28.4 \\
\hline 8 & $M$ & 51 & $R / L$ & $\mathrm{n} / \mathrm{d}$ & $\mathrm{n} / \mathrm{d}$ & $\mathrm{n} / \mathrm{d}$ & 29.9 \\
\hline 9 & $M$ & 56 & $\mathrm{R}$ & HT & $\mathrm{n} / \mathrm{d}$ & $S>10$ & 22.8 \\
\hline 10 & $M$ & 76 & $R / L$ & $\mathrm{n} / \mathrm{d}$ & $\mathrm{n} / \mathrm{d}$ & $\mathrm{n} / \mathrm{d}$ & 30.2 \\
\hline II & M & 49 & $R / L$ & $\mathrm{n} / \mathrm{d}$ & $n / d$ & $\mathrm{~S}<10$ & 27.1 \\
\hline 12 & $M$ & 56 & $\mathrm{~L}$ & HT & $\mathrm{n} / \mathrm{d}$ & $n / d$ & 28.1 \\
\hline 13 & M & 84 & $R / L$ & $\mathrm{n} / \mathrm{d}$ & $\mathrm{n} / \mathrm{d}$ & $S>10$ & 28.4 \\
\hline 14 & $M$ & 59 & $R / L$ & $\mathrm{n} / \mathrm{d}$ & DM & $\mathrm{n} / \mathrm{d}$ & 29.3 \\
\hline 15 & $M$ & 71 & $R / L$ & HT & $\mathrm{n} / \mathrm{d}$ & $S>10$ & 29.3 \\
\hline 16 & $M$ & 53 & $R / L$ & $\mathrm{n} / \mathrm{d}$ & $\mathrm{n} / \mathrm{d}$ & $\mathrm{S}<10$ & 27.1 \\
\hline 17 & $M$ & 54 & $R / L$ & HT & DM & $S>10$ & 24.1 \\
\hline 18 & $M$ & 57 & $\mathrm{R}$ & $\mathrm{n} / \mathrm{d}$ & $\mathrm{n} / \mathrm{d}$ & $\mathrm{n} / \mathrm{d}$ & 29.7 \\
\hline 19 & $M$ & 65 & $R / L$ & HT & $\mathrm{n} / \mathrm{d}$ & $S>10$ & 29 \\
\hline 20 & $M$ & 47 & $R / L$ & $\mathrm{n} / \mathrm{d}$ & DM & $\mathrm{n} / \mathrm{d}$ & 27.5 \\
\hline 21 & $M$ & 48 & $R / L$ & $\mathrm{n} / \mathrm{d}$ & $\mathrm{n} / \mathrm{d}$ & $\mathrm{S}<10$ & 30.7 \\
\hline 22 & $M$ & 64 & $\mathrm{R}$ & HT & DM & $\mathrm{n} / \mathrm{d}$ & 27.1 \\
\hline 23 & $M$ & 74 & $R / L$ & $\mathrm{n} / \mathrm{d}$ & $\mathrm{n} / \mathrm{d}$ & $\mathrm{n} / \mathrm{d}$ & 22.8 \\
\hline 24 & $M$ & 65 & $R / L$ & $\mathrm{n} / \mathrm{d}$ & $\mathrm{n} / \mathrm{d}$ & $\mathrm{S}<10$ & 19.8 \\
\hline 25 & $M$ & 64 & $\mathrm{~L}$ & HT & $\mathrm{n} / \mathrm{d}$ & $\mathrm{n} / \mathrm{d}$ & 28.8 \\
\hline 26 & $M$ & 54 & $R / L$ & $\mathrm{n} / \mathrm{d}$ & $\mathrm{n} / \mathrm{d}$ & $\mathrm{n} / \mathrm{d}$ & 30.5 \\
\hline 27 & $M$ & 45 & $R / L$ & $\mathrm{n} / \mathrm{d}$ & $\mathrm{n} / \mathrm{d}$ & $\mathrm{S}<10$ & 33 \\
\hline 28 & $M$ & 57 & $\mathrm{R}$ & $\mathrm{HT}$ & $\mathrm{n} / \mathrm{d}$ & $\mathrm{n} / \mathrm{d}$ & 24.2 \\
\hline 29 & $\mathrm{~F}$ & 59 & $\mathrm{R}$ & $\mathrm{n} / \mathrm{d}$ & $\mathrm{n} / \mathrm{d}$ & $\mathrm{n} / \mathrm{d}$ & 28.3 \\
\hline 30 & $\mathrm{~F}$ & 75 & $\mathrm{R}$ & HT & $\mathrm{n} / \mathrm{d}$ & $\mathrm{n} / \mathrm{d}$ & 29 \\
\hline 31 & $\mathrm{~F}$ & 55 & $\mathrm{~L}$ & $\mathrm{n} / \mathrm{d}$ & DM & $n / d$ & 27.5 \\
\hline 32 & $\mathrm{~F}$ & 59 & $\mathrm{R}$ & $\mathrm{n} / \mathrm{d}$ & $\mathrm{n} / \mathrm{d}$ & $\mathrm{n} / \mathrm{d}$ & 27.9 \\
\hline 33 & $\mathrm{~F}$ & 59 & $\mathrm{~L}$ & HT & $\mathrm{n} / \mathrm{d}$ & $\mathrm{n} / \mathrm{d}$ & 22.6 \\
\hline 34 & $\mathrm{~F}$ & 62 & $\mathrm{R}$ & $\mathrm{n} / \mathrm{d}$ & $\mathrm{n} / \mathrm{d}$ & $\mathrm{n} / \mathrm{d}$ & 31.6 \\
\hline 35 & $\mathrm{~F}$ & 46 & $\mathrm{~L}$ & $\mathrm{n} / \mathrm{d}$ & DM & $\mathrm{n} / \mathrm{d}$ & 25.3 \\
\hline 36 & $\mathrm{~F}$ & 58 & $\mathrm{R}$ & HT & DM & $\mathrm{n} / \mathrm{d}$ & 26.4 \\
\hline 37 & $\mathrm{~F}$ & 71 & $\mathrm{~L}$ & $\mathrm{n} / \mathrm{d}$ & $\mathrm{n} / \mathrm{d}$ & $\mathrm{n} / \mathrm{d}$ & 23.8 \\
\hline 38 & $\mathrm{~F}$ & 81 & $R / L$ & $\mathrm{n} / \mathrm{d}$ & DM & $n / d$ & 21.5 \\
\hline 39 & $\mathrm{~F}$ & 58 & $\mathrm{~L}$ & HT & $\mathrm{n} / \mathrm{d}$ & $\mathrm{n} / \mathrm{d}$ & 25.2 \\
\hline 40 & $\mathrm{~F}$ & 61 & $R / L$ & $\mathrm{n} / \mathrm{d}$ & DM & $\mathrm{n} / \mathrm{d}$ & 29.3 \\
\hline $4 I$ & $\mathrm{~F}$ & 53 & $\mathrm{R}$ & $\mathrm{n} / \mathrm{d}$ & $\mathrm{n} / \mathrm{d}$ & $\mathrm{n} / \mathrm{d}$ & 27.4 \\
\hline 42 & $\mathrm{~F}$ & 46 & $R / L$ & $\mathrm{n} / \mathrm{d}$ & $\mathrm{n} / \mathrm{d}$ & $\mathrm{n} / \mathrm{d}$ & 25.7 \\
\hline 43 & $\mathrm{~F}$ & 49 & $\mathrm{R}$ & HT & $\mathrm{n} / \mathrm{d}$ & $S>10$ & 25.5 \\
\hline 44 & $\mathrm{~F}$ & 69 & $\mathrm{R}$ & $\mathrm{n} / \mathrm{d}$ & $\mathrm{n} / \mathrm{d}$ & $\mathrm{n} / \mathrm{d}$ & 25.2 \\
\hline 45 & $\mathrm{~F}$ & 76 & $R / L$ & $\mathrm{n} / \mathrm{d}$ & $\mathrm{n} / \mathrm{d}$ & $\mathrm{n} / \mathrm{d}$ & 26.2 \\
\hline 46 & $\mathrm{~F}$ & 46 & $\mathrm{R}$ & $\mathrm{HT}$ & DM & $\mathrm{n} / \mathrm{d}$ & 21.8 \\
\hline 47 & $\mathrm{~F}$ & 54 & $\mathrm{R}$ & $\mathrm{n} / \mathrm{d}$ & $\mathrm{n} / \mathrm{d}$ & $\mathrm{n} / \mathrm{d}$ & 23 \\
\hline 48 & $\mathrm{~F}$ & 49 & $\mathrm{~L}$ & $\mathrm{n} / \mathrm{d}$ & DM & $\mathrm{n} / \mathrm{d}$ & 27.1 \\
\hline 49 & $\mathrm{~F}$ & 52 & $R / L$ & $\mathrm{n} / \mathrm{d}$ & $\mathrm{n} / \mathrm{d}$ & $\mathrm{n} / \mathrm{d}$ & 26.9 \\
\hline 50 & $\mathrm{~F}$ & 65 & $R / L$ & HT & $\mathrm{n} / \mathrm{d}$ & $\mathrm{n} / \mathrm{d}$ & 25.6 \\
\hline 51 & $\mathrm{~F}$ & 78 & $\mathrm{R}$ & $\mathrm{n} / \mathrm{d}$ & $\mathrm{n} / \mathrm{d}$ & $\mathrm{n} / \mathrm{d}$ & 32 \\
\hline 52 & $\mathrm{~F}$ & 64 & $R / L$ & $\mathrm{n} / \mathrm{d}$ & DM & $\mathrm{n} / \mathrm{d}$ & 30.1 \\
\hline 53 & $\mathrm{~F}$ & 70 & $\mathrm{~L}$ & $\mathrm{HT}$ & $\mathrm{n} / \mathrm{d}$ & $\mathrm{n} / \mathrm{d}$ & 25 \\
\hline 54 & $\mathrm{~F}$ & 65 & $R / L$ & $\mathrm{n} / \mathrm{d}$ & $\mathrm{n} / \mathrm{d}$ & $\mathrm{n} / \mathrm{d}$ & 31.2 \\
\hline 55 & $\mathrm{~F}$ & 80 & $\mathrm{R}$ & $\mathrm{n} / \mathrm{d}$ & DM & $\mathrm{S}<10$ & 25.8 \\
\hline 56 & $\mathrm{~F}$ & 54 & $\mathrm{~L}$ & $\mathrm{n} / \mathrm{d}$ & $\mathrm{n} / \mathrm{d}$ & $\mathrm{n} / \mathrm{d}$ & 20 \\
\hline 57 & $\mathrm{~F}$ & 46 & $R / L$ & $\mathrm{n} / \mathrm{d}$ & $\mathrm{n} / \mathrm{d}$ & $\mathrm{n} / \mathrm{d}$ & 26.7 \\
\hline 58 & $\mathrm{~F}$ & 81 & $R / L$ & HT & $\mathrm{n} / \mathrm{d}$ & $\mathrm{n} / \mathrm{d}$ & 24.8 \\
\hline 59 & $\mathrm{~F}$ & 72 & $\mathrm{~L}$ & $\mathrm{n} / \mathrm{d}$ & DM & $\mathrm{n} / \mathrm{d}$ & 26.7 \\
\hline
\end{tabular}

Abbreviations: $M$, male; $F$, female; R, calcified carotid atheroma at right carotid; L, calcified carotid atheroma at left carotid; R/L, calcified carotid atheroma at right and left carotid; HT, hypertension; DM, diabetes mellitus; BMI, body mass index; $\mathrm{n} / \mathrm{d}$, not detected; $\mathrm{S}>10$, smoking more than 10 cigarettes daily; $\mathrm{S}<\mathrm{I0}$, smoking less than 10 cigarettes daily. 


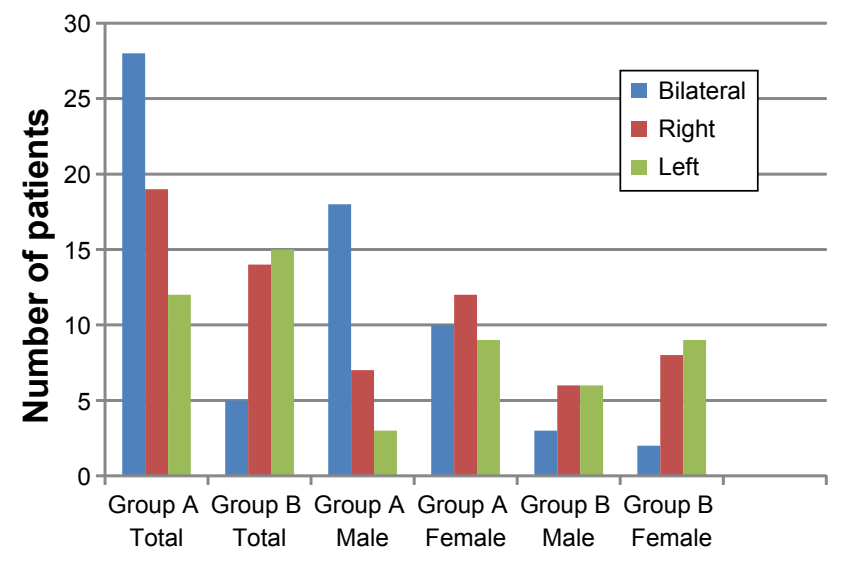

Figure 5 Numerical distribution of radiographic findings of CCA according to sex and side.

Note: Group A (study group) and Group B (control group)

Abbreviation: CCA, calcified carotid atheroma.

older population. Group A was defined as the study group with findings confirmed by DU and Group B as the control group with findings were not confirmed by DU.

Friedlander and Lande $^{7}$ first found the incidence of CCA on PRs and suggested that the panoramic image may be a helpful in detecting patients at risk of stroke. Since the first report, many studies have been carried out to determine the incidence of CCA on PRs ${ }^{5,8,16-22}$ and to verify the usefulness of the PR in the identification of stoke-prone patients through correlation with medical history and Doppler spectral analysis. ${ }^{5,11,12,16-18}$

DU is a method of examining vascular structures with sound waves. It provides hemodynamic information about the carotid and vertebral arteries. Despite sensitivity of $92.6 \%$ and specificity of $97 \%$, angiography is accepted as the gold standard. ${ }^{23}$ In the current study, DU was used for

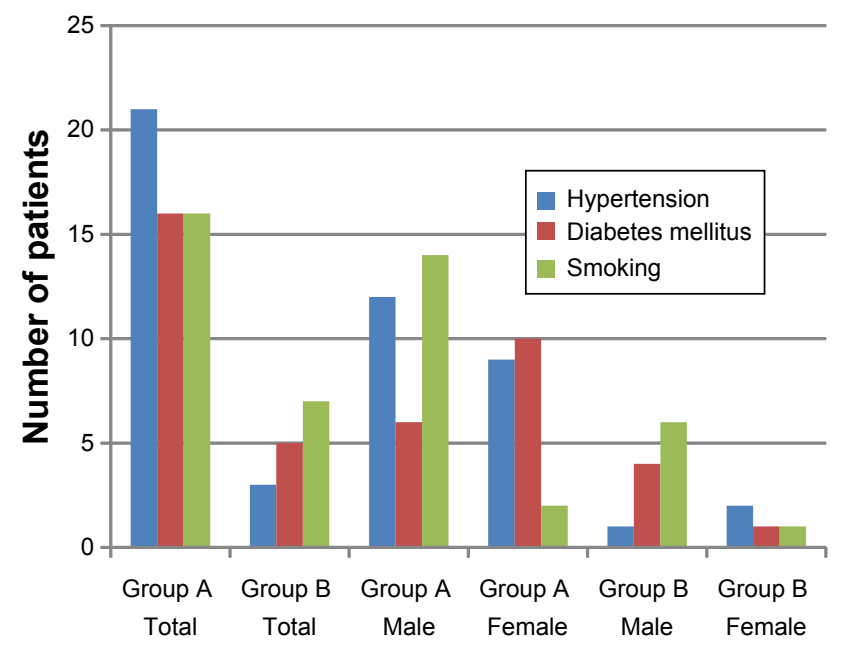

Figure 6 Numerical distribution of hypertension, diabetes mellitus, and smoking in Group A (study group) and Group B (control group) according to sex.
Table 2 The demographic and medical records of Group B

\begin{tabular}{|c|c|c|c|c|c|c|c|}
\hline Patient & Sex & $\begin{array}{l}\text { Age } \\
\text { (years) }\end{array}$ & Side & HT & DM & Smoking & $\begin{array}{l}\text { BMI } \\
\left(\mathrm{kg} / \mathrm{m}^{2}\right)\end{array}$ \\
\hline I & $M$ & 64 & $\mathrm{R}$ & $\mathrm{n} / \mathrm{d}$ & DM & $\mathrm{n} / \mathrm{d}$ & 27.7 \\
\hline 2 & $M$ & 65 & $\mathrm{~L}$ & $\mathrm{n} / \mathrm{d}$ & $\mathrm{n} / \mathrm{d}$ & $\mathrm{S}<10$ & 32.2 \\
\hline 3 & $M$ & 47 & $\mathrm{R}$ & $\mathrm{n} / \mathrm{d}$ & $\mathrm{n} / \mathrm{d}$ & $\mathrm{n} / \mathrm{d}$ & 29.7 \\
\hline 4 & $M$ & 64 & $\mathrm{~L}$ & $\mathrm{n} / \mathrm{d}$ & $\mathrm{n} / \mathrm{d}$ & $\mathrm{n} / \mathrm{d}$ & 28 \\
\hline 5 & $M$ & 45 & $\mathrm{R}$ & $\mathrm{n} / \mathrm{d}$ & $\mathrm{n} / \mathrm{d}$ & $\mathrm{n} / \mathrm{d}$ & 25.5 \\
\hline 6 & $M$ & 62 & $\mathrm{~L}$ & $\mathrm{n} / \mathrm{d}$ & $\mathrm{n} / \mathrm{d}$ & $\mathrm{n} / \mathrm{d}$ & 27.8 \\
\hline 7 & $M$ & 47 & $R / L$ & $\mathrm{n} / \mathrm{d}$ & $\mathrm{n} / \mathrm{d}$ & $\mathrm{n} / \mathrm{d}$ & 25.5 \\
\hline 8 & $M$ & 63 & $\mathrm{~L}$ & $\mathrm{n} / \mathrm{d}$ & $\mathrm{n} / \mathrm{d}$ & $S<10$ & 29.9 \\
\hline 9 & $M$ & 46 & $\mathrm{R}$ & $\mathrm{n} / \mathrm{d}$ & $\mathrm{n} / \mathrm{d}$ & $\mathrm{n} / \mathrm{d}$ & 24.7 \\
\hline 10 & $M$ & 49 & $\mathrm{~L}$ & $\mathrm{n} / \mathrm{d}$ & $\mathrm{n} / \mathrm{d}$ & $S>10$ & 29.3 \\
\hline 11 & $M$ & 65 & $R / L$ & $\mathrm{n} / \mathrm{d}$ & $\mathrm{n} / \mathrm{d}$ & $\mathrm{n} / \mathrm{d}$ & 22.5 \\
\hline 12 & $M$ & 54 & $\mathrm{R}$ & $\mathrm{n} / \mathrm{d}$ & $\mathrm{n} / \mathrm{d}$ & $S>10$ & 23.9 \\
\hline 13 & $M$ & 68 & $R / L$ & $\mathrm{n} / \mathrm{d}$ & $\mathrm{n} / \mathrm{d}$ & $S>10$ & 30.7 \\
\hline 14 & $M$ & 67 & $\mathrm{R}$ & $\mathrm{HT}$ & $\mathrm{n} / \mathrm{d}$ & $\mathrm{n} / \mathrm{d}$ & 32.4 \\
\hline 15 & $M$ & 49 & $\mathrm{~L}$ & $\mathrm{n} / \mathrm{d}$ & $\mathrm{n} / \mathrm{d}$ & $s>10$ & 33.6 \\
\hline 16 & $\mathrm{~F}$ & 48 & $R / L$ & $\mathrm{n} / \mathrm{d}$ & $\mathrm{n} / \mathrm{d}$ & $\mathrm{n} / \mathrm{d}$ & 24.4 \\
\hline 17 & $F$ & 47 & $\mathrm{R}$ & $\mathrm{n} / \mathrm{d}$ & $\mathrm{n} / \mathrm{d}$ & $\mathrm{n} / \mathrm{d}$ & 22 \\
\hline 18 & $\mathrm{~F}$ & 83 & $\mathrm{~L}$ & $\mathrm{n} / \mathrm{d}$ & $\mathrm{n} / \mathrm{d}$ & $\mathrm{n} / \mathrm{d}$ & 23.4 \\
\hline 19 & $\mathrm{~F}$ & 56 & $\mathrm{R}$ & $\mathrm{n} / \mathrm{d}$ & $\mathrm{n} / \mathrm{d}$ & $\mathrm{n} / \mathrm{d}$ & 28.2 \\
\hline 20 & $\mathrm{~F}$ & 49 & $\mathrm{~L}$ & $\mathrm{n} / \mathrm{d}$ & $\mathrm{n} / \mathrm{d}$ & $\mathrm{n} / \mathrm{d}$ & 23.8 \\
\hline 21 & $F$ & 62 & $R / L$ & HT & $\mathrm{n} / \mathrm{d}$ & $\mathrm{n} / \mathrm{d}$ & 29.4 \\
\hline 22 & $\mathrm{~F}$ & 57 & $\mathrm{~L}$ & $\mathrm{n} / \mathrm{d}$ & $\mathrm{n} / \mathrm{d}$ & $\mathrm{n} / \mathrm{d}$ & 25.7 \\
\hline 23 & $\mathrm{~F}$ & 49 & $\mathrm{R}$ & $\mathrm{n} / \mathrm{d}$ & $\mathrm{n} / \mathrm{d}$ & $\mathrm{n} / \mathrm{d}$ & 21.8 \\
\hline 24 & $\mathrm{~F}$ & 48 & $\mathrm{R}$ & $\mathrm{n} / \mathrm{d}$ & $\mathrm{n} / \mathrm{d}$ & $\mathrm{n} / \mathrm{d}$ & 23.8 \\
\hline 25 & $F$ & 71 & $\mathrm{~L}$ & $\mathrm{n} / \mathrm{d}$ & $\mathrm{n} / \mathrm{d}$ & $\mathrm{n} / \mathrm{d}$ & 27.9 \\
\hline 26 & $\mathrm{~F}$ & 51 & $\mathrm{~L}$ & $\mathrm{n} / \mathrm{d}$ & DM & $\mathrm{n} / \mathrm{d}$ & 27.1 \\
\hline 27 & $F$ & 65 & $\mathrm{~L}$ & $\mathrm{n} / \mathrm{d}$ & $\mathrm{n} / \mathrm{d}$ & $\mathrm{S}<10$ & 26.8 \\
\hline 28 & $F$ & 73 & $\mathrm{~L}$ & $\mathrm{n} / \mathrm{d}$ & $\mathrm{n} / \mathrm{d}$ & $\mathrm{n} / \mathrm{d}$ & 26.5 \\
\hline 29 & $\mathrm{~F}$ & 57 & $\mathrm{R}$ & $\mathrm{n} / \mathrm{d}$ & $\mathrm{n} / \mathrm{d}$ & $\mathrm{n} / \mathrm{d}$ & 23.8 \\
\hline 30 & $F$ & 68 & $\mathrm{~L}$ & $\mathrm{n} / \mathrm{d}$ & $\mathrm{n} / \mathrm{d}$ & $\mathrm{n} / \mathrm{d}$ & 26.7 \\
\hline 31 & $\mathrm{~F}$ & 45 & $\mathrm{R}$ & $\mathrm{n} / \mathrm{d}$ & $\mathrm{n} / \mathrm{d}$ & $\mathrm{n} / \mathrm{d}$ & 29.4 \\
\hline 32 & $F$ & 83 & $R$ & HT & $\mathrm{n} / \mathrm{d}$ & $\mathrm{n} / \mathrm{d}$ & 31 \\
\hline 33 & $\mathrm{~F}$ & 74 & $\mathrm{R}$ & $\mathrm{n} / \mathrm{d}$ & $\mathrm{n} / \mathrm{d}$ & $\mathrm{n} / \mathrm{d}$ & 28 \\
\hline 34 & $F$ & 57 & $\mathrm{R}$ & $\mathrm{n} / \mathrm{d}$ & $\mathrm{n} / \mathrm{d}$ & $\mathrm{n} / \mathrm{d}$ & 23.9 \\
\hline
\end{tabular}

Abbreviations: $\mathrm{M}$, male; F, female; R, calcified carotid atheroma at right carotid; L, calcified carotid atheroma at left carotid; R/L, calcified carotid atheroma at right and left carotid; $H T$, hypertension; DM, diabetes mellitus; BMI, body mass index; $n / d$, not detected; $\mathrm{S}>10$, smoking more than 10 cigarettes daily; $\mathrm{S}<10$, smoking less than 10 cigarettes daily.

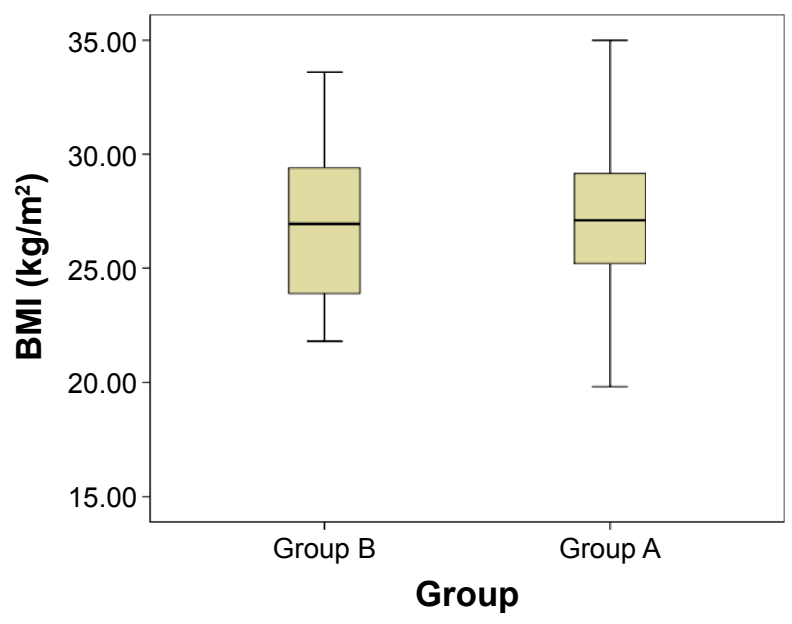

Figure 7 Comparison of BMI between Group A and Group B.

Note: Group A (study group) and Group B (control group). Abbreviation: BMI, body mass index. 


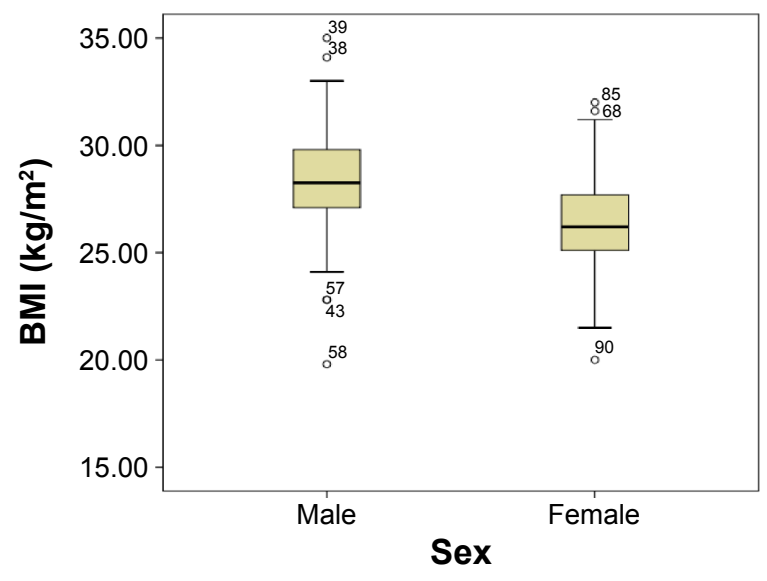

Figure 8 Comparison of BMI in Group A according to sex.

Note: Group A (study group).

Abbreviation: BMI, body mass index.

confirmation of CCA determined on PRs as it is a noninvasive method.

In our study, the diagnostic accuracy of PR for the detection of CCA was evaluated using DU. The results of this study showed $63.44 \%$ accuracy for the detection of CCA using PR. In a study by Yoon et $\mathrm{al}^{24}$ similar accuracy was observed at $62.3 \%$. In other studies, Baumann-Bhalla et $\mathrm{al}^{25}$ and Almog et $\mathrm{al}^{26}$ found the calcification rate with sonographic examination in atherosclerotic patients to be $81.5 \%$ and $65 \%$, respectively. In the general population, the prevalence of CCA detected on PRs varies from $0.43 \%$ to $5 \%$ depending on age, sex, ethnicity, and lifestyle..$^{9,16,17,26-29}$ In the current study, a similar result of $5.63 \%$ CCA was determined in the general population. CCAs rates were not significantly different between males and females $(P=0.756)$. The same finding of no difference in the male-female rates was reported by Ohba et al, ${ }^{29}$ Bayram et al. ${ }^{30}$ However, Tamura et al ${ }^{31}$ identified a significant difference on PRs between the male and female rates. We thought

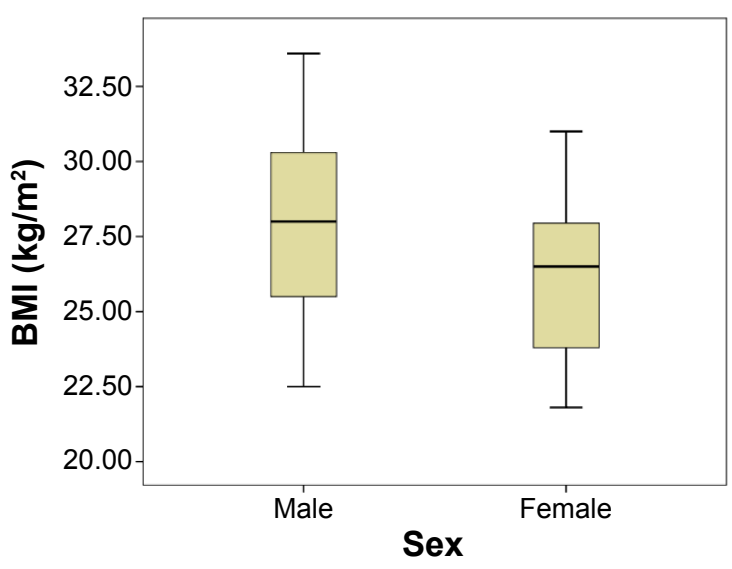

Figure 9 Comparison of BMI in Group B according to sex.

Note: Group B (control group).

Abbreviation: BMI, body mass index. that all these different CCA prevalences in female and male populations were probably due to ethnicity, eating habits, or social life.

In the present study in Group A and Group B, presumed CCA was identified on the left or right side, and no significant statistical differences were determined between the two sides. This finding differs from other studies, as in the majority of previous reports of normal populations, ${ }^{8,16,31} \mathrm{CCA}$ has been observed to be unilateral and located on the left side rather than the right side. However, Şişman et $\mathrm{al}^{32}$ and Ohba et $\mathrm{al}^{29}$ demonstrated CCA to be generally located on the right side. These differences in CCA localizations in the reports may be related to the study samples.

Studies showed that atherosclerotic lesions should be verified radiologically by a further diagnostic tool. ${ }^{33,34}$ It is thought that the reason for large differences in the CCA prevalence rates determined on PRs could be attributed to not making a differential diagnosis of the anatomic formations in the evaluation of the panoramic films. Several formations can appear as radiopacities in this region of the PR, including salivary calculi, calcified lymphoid tissue, tonsilloliths, the stylohyoid complex, and the triticeous cartilage. ${ }^{35}$ False negatives may also be associated with the detection of CCA on PRs as the CCA may be inferior to the area covered by the PR or, if it is within this area, may not be visible because the degree of calcification is insufficient to be seen. ${ }^{36}$

The sorting of obesity and overweight according to BMI by WHO is intended for international utilization. They show risk for type 2 diabetes and cardiovascular diseases, which are quickly becoming main reasons of death in adults. ${ }^{14}$ In the present study, in the comparisons of Group A with Group $\mathrm{B}$, no correlation was determined between CCA and BMI. This is consistent with the results of Irace et $\mathrm{al}^{37}$ and Ertas and $\operatorname{Sisman}^{38}$ who reported that an increase in body weight is not associated with prevalence or degree of CCA. However, Carter et al found that obesity was most strongly related to the occurrence of CCA. ${ }^{16}$ People with type 2 diabetes mellitus are disproportionately at risk of experiencing stroke, because hyperglycemia and other risk factors associated with diabetes accelerate the development of CCA. In a study by Friedlander, individuals with type 2 diabetes were determined to have high rates of atheroma visualized on PRs. ${ }^{22,39}$ The current study showed conflicting results with studies mentioned earlier, and there was no significant difference between Groups A and B in respect of diabetes mellitus. Especially, hypertension is one of the most important factors in atheroma formation and is crucial in patients with CCA. ${ }^{40}$ In a study by Johansson et $\mathrm{al}^{34}$ there was no significant difference between the group 
with stenosis and the group without stenosis in hypertensive patients. Pornprasertsuk-Damrongsri and Thanakun ${ }^{8}$ found that patients with CCA were associated with hypertension $(52.9 \%)$. Cohen et $\mathrm{al}^{5}$ revealed that $53.5 \%$ of patients with CCA had a history of hypertension. In the current study, hypertension rates were found to be four times higher in Group A (35.59\%) than in Group B (8.82\%), and also was statistically significant. These conflicting results regarding CCA and systemic findings may be partly related to the size of samples in the studies or may be due to regional differences, lifestyle, and dietary factors.

In many epidemiological studies reported in literatures, smoking has been noted to be a major risk factor of cerebral infarction. ${ }^{41-43}$ The current study found that CCA was not affected by smoking, although in both groups, a significant difference was determined between the male and female population. We considered that decreased CCA prevalence in smoker persons are probably due to the amount of daily smoking or due to the less number of smoker individuals who participated in our study.

There were some limitations to the present study. Further study might be needed to clarify the clinical significance between PR and DU. No analysis was made of the appearance (intensity, size, and/or shape) of the calcification in the area of the carotid arteries on the panoramic images. These factors might be useful to select individuals for further carotid screening. Another limitation of the present study was the small number of subjects. Hypercholesterolemia could have been analyzed but that would have involved the invasive procedure of blood sampling and the associated ethical restrictions. The PRs in this study could have been evaluated by an oral and maxillofacial radiologist. However, as PRs are now found in nearly all dental clinics, the images are evaluated by dental practitioners. Therefore, it was aimed to provide an evaluation by physicians, not oral and maxillofacial radiologists.

\section{Conclusion}

Almost all patients undergo PR as part of their routine dental examinations and treatments. ${ }^{44-46}$ Although PR cannot be used as an initial diagnostic method when searching for CCA, dentists should be aware of CCA on routine PR, particularly in older patients who may also have the risk factors of obesity, diabetes mellitus, hypertension, and smoking. Recognition of CCA, especially in hypertensive patients, could potentially increase the length and quality of life for individuals. Proper findings of CCA and early referral for investigation could potentially decrease morbidity and mortality in the population. A medical consultation should be requested for all patients who present with CCA on PRs.

\section{Acknowledgment}

We would like to thank Dr Ismet Dogan for his support in statistical analyses.

\section{Disclosure}

The authors report no conflicts of interest in this work.

\section{References}

1. Williams GR, Jiang JG, Matchar DB, Samsa GP. Incidence and occurrence of total (first-ever and recurrent) stroke. Stroke. 1999;30:2523-2528.

2. Wakhloo AK, Lieber BB, Seong J, et al. Hemodynamics of carotid artery atherosclerotic occlusive disease. J Vasc Interv Radiol. 2004;15: S111-S121.

3. Levy C, Mandel L. Calcified carotid artery imaged by computed tomography. Int J Oral Maxillofac Surg. 2010;68:218-220.

4. Carter LC, Tsimidis K, Fabiano J. Carotid calcifications on panoramic radiography identify an asymptomatic male patient at risk for stroke: a case report. Oral Surg Oral Med Oral Pathol Oral Radiol Endod. 1998;85:119-122.

5. Cohen SN, Friedlander AH, Jolly DA, Date L. Carotid calcification on panoramic radiographs: an important marker for vascular risk. Oral Surg Oral Med Oral Pathol Oral Radiol Endod. 2002;94:510-514.

6. Bayer S, Helfgen E-H, Bös C, Kraus D, Enkling N, Mues S. Prevalence of findings compatible with carotid artery calcifications on dental panoramic radiographs. Clin Oral Investig. 2011;15:563-569.

7. Friedlander AH, Lande A. Panoramic radiographic identification of carotid arterial plaques. Oral Surg Oral Med Oral Pathol Oral Radiol Endod. 1981;52:102-104

8. Pornprasertsuk-Damrongsri S, Thanakun S. Carotid artery calcification detected on panoramic radiographs in a group of Thai population. Surg Oral Med Oral Pathol Oral Radiol Endod. 2006;101:110-115.

9. Kumagai M, Yamagishi T, Fukui N, Chiba M. Carotid artery calcification seen on panoramic dental radiographs in the Asian population in Japan. Dentomaxillofac Radiol. 2007;36:92-96.

10. Manzi FR, Bóscolo FN, Almeida SMD, Neto FH. Panoramic radiography as an auxiliary in detecting patients at risk for cerebrovascular accident (CVA): a case report. J Oral Rehabil. 2003;45:177-180.

11. Friedlander AH, Gratt BM. Panoramic dental radiography as an aid in detecting patients at risk for stroke. J Oral Maxillofac Surg. 1994; 52:1257-1262.

12. Friedlander A. Identification of stroke-prone patients by panoramic and cervical spine radiography. Dentomaxillofacial Radiol. 1995;24:160-164

13. Christou P, Leemann B, Schimmel M, Kiliaridis S, Müller F. Carotid artery calcification in ischemic stroke patients detected in standard dental panoramic radiographs-a preliminary study. Adv Med Sci. 2010; 55:26-31.

14. Tan K. Appropriate body-mass index for Asian populations and its implications for policy and intervention strategies. The Lancet. 2004; 363:157-163.

15. Friedlander AH. Panoramic radiography: the differential diagnosis of carotid artery atheromas. Spec Care Dentist. 1995;15:223-227.

16. Carter LC, Haller AD, Nadarajah V, Calamel AD, Aguirre A. Use of panoramic radiography among an ambulatory dental population to detect patients at risk of stroke. J Am Dent Assoc. 1997;128:977-984.

17. Friedlander AH, Baker JD. Panoramic radiography: an aid in detecting patients at risk of cerebrovascular accident. J Am Dent Assoc. 1994; 125:1598-1603. 
18. Friedlander AH, Manesh F, Wasterlain CG. Prevalence of detectable carotid artery calcifications on panoramic radiographs of recent stroke victims. Oral Surg Oral Med Oral Pathol Oral Radiol Endod. 1994;77:669-673.

19. Friedlander AH, Friedlander IK, Yueh R, Littner MR. The prevalence of carotid atheromas seen on panoramic radiographs of patients with obstructive sleep apnea and their relation to risk factors for atherosclerosis. J Oral Maxillofac Surg. 1999;57:516-521.

20. Freymiller E, Sung E, Friedlander A. Detection of radiation-induced cervical atheromas by panoramic radiography. Oral Oncol. 2000;36: 175-179.

21. Sung EC, Friedlander AH, Kobashigawa JA. The prevalence of calcified carotid atheromas on the panoramic radiographs of patients with dilated cardiomyopathy. Oral Surg Oral Med Oral Pathol Oral Radiol Endod. 2004;97:404-407.

22. Friedlander AH, Maeder LA. The prevalence of calcified carotid artery atheromas on the panoramic radiographs of patients with type 2 diabetes mellitus. Oral Surg Oral Med Oral Pathol Oral Radiol Endod. 2000;89:420-424.

23. Yurdakul S, Aytekin S. Doppler ultrasound imaging of the carotid and vertebral arteries. Turk Kardiyol Dern Ars. 2011;39:508-517.

24. Yoon S, Yoon W, Kim O, Lee J, Kang B. Diagnostic accuracy of panoramic radiography in the detection of calcified carotid artery. Dentomaxillofac Radiol. 2008;37(2):104-108.

25. Baumann-Bhalla S, Meier R, Burow A, et al. Recognizing calcifications of the carotid artery on panoramic radiographs to prevent strokes. Schweiz Monatsschr Zahnmed. 2012;122(11):1016-1029.

26. Almog DM, Horev T, Illig KA, Green RM, Carter LC. Correlating carotid artery stenosis detected by panoramic radiography with clinically relevant carotid artery stenosis determined by duplex ultrasound. Oral Surg Oral Med Oral Pathol Oral Radiol Endod. 2002;94: 768-773.

27. Lewis D, Brooks S. Cartoid artery calcification in a general dental population: a retrospective study of panoramic radiographs. Gen Dent. 1998;47:98-103.

28. Hubar J. Carotid artery calcification in the black population: a retrospective study on panoramic radiographs. Dentomaxillofac Radiol. 1999;28:348-350.

29. Ohba T, Takata Y, Ansai T, et al. Evaluation of calcified carotid artery atheromas detected by panoramic radiograph among 80 -year-olds. Oral Surg Oral Med Oral Pathol Oral Radiol Endod. 2003;96:647-650.

30. Bayram B, Uckan S, Acikgoz A, Müderrisoğlu H, Aydinalp A. Digital panoramic radiography: a reliable method to diagnose carotid artery atheromas? Dento maxillo facial radiology. 2006;35(4):266-270.

31. Tamura $T$, Inui $M$, Nakase $M$, Nakamura $S$, Okumura $K$, Tagawa $T$. Clinicostatistical study of carotid calcification on panoramic radiographs. Oral Dis. 2005;11:314-317.
32. Sisman Y, Ertas ET, Gokce C, Menku A, Ulker M, Akgunlu F. The prevalence of carotid artery calcification on the panoramic radiographs in Cappadocia Region population. Eur J Dent. 2007;1:132.

33. Damaskos S, Griniatsos J, Tsekouras N, et al. Reliability of panoramic radiograph for carotid atheroma detection: a study in patients who fulfill the criteria for carotid endarterectomy. Oral Surg Oral Med Oral Pathol Oral Radiol Endod. 2008;106:736-742.

34. Johansson EP, Ahlqvist J, Garoff M, Karp K, Jäghagen EL, Wester P. Ultrasound screening for asymptomatic carotid stenosis in subjects with calcifications in the area of the carotid arteries on panoramic radiographs: a cross-sectional study. BMC Cardiovasc Disord. 2011; $11: 44$.

35. MacDonald D. Oral and Maxillofacial Radiology: A Diagnostic Approach. Ames, IA: Wiley-Blackwell; 2011.

36. Friedlander AH. Odd practice. Br Dent J. 2009;207:464-465.

37. Irace C, Scavelli F, Carallo C, Serra R, Cortese C, Gnasso A. Body mass index, metabolic syndrome and carotid atherosclerosis. Coron Artery Dis. 2009;20:94-99.

38. Ertas ET, Sisman Y. Detection of incidental carotid artery calcifications during dental examinations: panoramic radiography as an important aid in dentistry. Oral Surg Oral Med Oral Pathol Oral Radiol Endod. 2011;112:e11-e17.

39. Friedlander AH, Garrett NR, Norman DC. The prevalence of calcified carotid artery atheromas on the panoramic radiographs of patients with type 2 diabetes mellitus. J Am Dent Assoc. 2002;133:1516-1523.

40. MacDonald D, Chan A, Harris A, Vertinsky T, Farman AG, Scarfe WC. Diagnosis and management of calcified carotid artery atheroma: dental perspectives. Oral Surg Oral Med Oral Pathol Oral Radiol. 2012; 114:533-547.

41. Ueshima H, Choudhury SR, Okayama A, et al. Cigarette smoking as a risk factor for stroke death in Japan NIPPON DATA80. Stroke. 2004;35:1836-1841.

42. Abbott RD, Yin Y, Reed DM, Yano K. Risk of stroke in male cigarette smokers. N Engl J Med. 1986;315:717-720.

43. Nakayama T, Date C, Yokoyama T, Yoshiike N, Yamaguchi M, Tanaka H. A 15. 5-year follow-up study of stroke in a Japanese Provincial City The Shibata Study. Stroke. 1997;28:45-52.

44. Council on Dental Materials I, Equipment. Recommendations in radiographic practices: an update, 1988. J Am Dent Assoc. 1989;118: 115-117.

45. Kogon S, Bohay R, Stephens R. A survey of the radiographic practices of general dentists for edentulous patients. Oral Surg Oral Med Oral Pathol Oral Radiol Endod. 1995;80:365-368.

46. Scandrett FR, Tebo HG, Miller JT, Quigley MB. Radiographic examination of the edentulous patient: Part I. Review of the literature and preliminary report comparing three methods. Oral Surg Oral Med Oral Pathol. 1973;35:266-274.
Clinical Interventions in Aging

\section{Publish your work in this journal}

Clinical Interventions in Aging is an international, peer-reviewed journal focusing on evidence-based reports on the value or lack thereof of treatments intended to prevent or delay the onset of maladaptive correlates of aging in human beings. This journal is indexed on PubMed Central, MedLine,

\section{Dovepress}

CAS, Scopus and the Elsevier Bibliographic databases. The manuscript management system is completely online and includes a very quick and fair peer-review system, which is all easy to use. Visit http://www.dovepress. com/testimonials.php to read real quotes from published authors. 